Check for updates

Cite this: Chem. Commun., 2021, 57, 4126

Received 19th February 2021 Accepted 17th March 2021

DOI: $10.1039 / \mathrm{d} 1 \mathrm{cc} 00950 \mathrm{~h}$

rsc.li/chemcomm

\section{Biaryl sulfonamides as cisoid azosteres for photopharmacology $\dagger$}

\author{
Piermichele Kobauri, (D) ${ }^{a}$ Wiktor Szymanski, (D) *b Fangyuan Cao, (D) ${ }^{c}$ \\ Sebastian Thallmair, (iD de Siewert J. Marrink, (iD d Martin D. Witte, iD ${ }^{f}$ \\ Frank J. Dekker (D) *c and Ben L. Feringa (D) *a
}

\begin{abstract}
Biaryl sulfonamides are excellent candidates for the azologization approach that yields photoswitchable drugs more active in their metastable cis state, compared to the stable trans state. Here we present the scope and limitations of this strategy for rational design in photopharmacology.
\end{abstract}

Photopharmacology employs light to control the bioactivity of drugs with exceptional bio-orthogonality and spatiotemporal precision, thus potentially increasing site- and organ-selectivity in pharmaceutical applications. ${ }^{1-4}$ The incorporation of molecular photoswitches ${ }^{5}$ into drugs enables this non-invasive control, since their irradiation induces reversible changes in the structure and properties of the drug. ${ }^{2}$ Because of favorable photochemical properties and ease of synthesis, azobenzene is the most common switch in photopharmacology. ${ }^{6}$ Its photoisomerization from the thermally stable trans-isomer to the metastable cis-isomer results in substantial changes in molecular shape (from planar to globular), end-to-end distance (from $9 \AA$ to $6 \AA$ ) and dipole moment (from $0 \mathrm{D}$ to $\sim 3 \mathrm{D}$ ). ${ }^{6}$

The design of photoswitchable bioactive compounds often relies on trial-and-error approaches and the assumption that photoinduced changes will inherently result in different

\footnotetext{
${ }^{a}$ Stratingh Institute for Chemistry, University of Groningen, Nijenborgh 4, Groningen, 9747 AG, The Netherlands. E-mail: b.l.feringa@rug.nl

${ }^{b}$ Medical Imaging Center, University of Groningen, University Medical Center Groningen, Hanzeplein 1, Groningen 9713 GZ, The Netherlands.

E-mail:w.szymanski@umcg.nl

${ }^{c}$ Chemical and Pharmaceutical Biology, Groningen Research Institute of Pharmacy, University of Groningen, A. Deusinglaan 1, Groningen, 9713 AV, The Netherlands. E-mail:f.j.dekker@rug.nl

${ }^{d}$ Groningen Biomolecular Sciences and Biotechnology Institute \& Zernike Institute for Advanced Materials, University of Groningen, Nijenborgh 7 , Groningen 9747 AG, The Netherlands

${ }^{e}$ Frankfurt Institute for Advanced Studies, Ruth-Moufang-Straße 1, Frankfurt am Main 60438, Germany

${ }^{f}$ Chemical Biology II, Stratingh Institute for Chemistry, University of Groningen, Groningen 9747 AG, The Netherlands

$\dagger$ Electronic supplementary information (ESI) available. See DOI: 10.1039/ d1 cc00950h
}

bioactivities. Nevertheless, two rational design strategies emerged in azobenzene-based photopharmacology: azo-extension and azologization. The former explores the structure-activity relationship (SAR) of existing drug scaffolds to identify suitable sites for the attachment of an azobenzene. ${ }^{4}$ In the latter approach, azobenzenes are introduced in the place of aromatic moieties that are sterically and electronically similar. Those privileged moieties were termed "azosteres". ${ }^{7}$ In most applications, ${ }^{8}$ photopharmacology aims to design photoswitchable drugs that are more active in the metastable state, i.e. "cis-on" in the case of classical azobenzenes. In particular, if this property is combined with a large difference in potency between the photoisomers, it ensures effective control of biological processes. ${ }^{1-3,8}$ However, most attempts at azologization resulted in "trans-on" ligands, especially for planar azosteres that adopt a transoid conformation (such as benzyl ether, ${ }^{7}$ benzanilide, ${ }^{10}$ and diphenylacetylene ${ }^{11}$ ). An alternative strategy, named "sign inversion", uses cyclic diazocines ${ }^{12}$ with thermally stable cis isomers, ${ }^{13}$ but their limited synthetic accessibility might hinder its applicability. ${ }^{14}$ Conversely, only a few examples of cisoid azosteres have been reported. ${ }^{9,15,16}$ Nevertheless, an explicit set of general criteria for the design of cis-on photoswitchable drugs is still missing, although they are expected to significantly expand the chemical space available for photopharmacology. Here we present the identification of the biaryl sulfonamide scaffold as a prevalent cisoid azostere, because of its structural and electronic similarity with cis-azobenzene.

First, we queried the Cambridge Structural Database (CSD) on the geometry of azobenzene and the most generic azostere, i.e., two (hetero)aromatic rings linked by two atoms. Measurement of dihedral and centroid angles, ring distances and ring angles provided insights into the requirements for a cisoid geometry (see ESI, $\uparrow$ Fig. S18), in particular the centroid angle being less than $100^{\circ}$ and the ring distance being less than $5 \AA$. Biaryl sulfonamides (BAS) emerged as cisoid scaffolds (Fig. 1A and B), in accordance with their known preference for a staggered conformation. ${ }^{17}$ This geometrical bias ${ }^{18}$ has been employed for conformational control in drug design. ${ }^{19}$ In addition to structural similarity to cis-azobenzene, electronic 
A
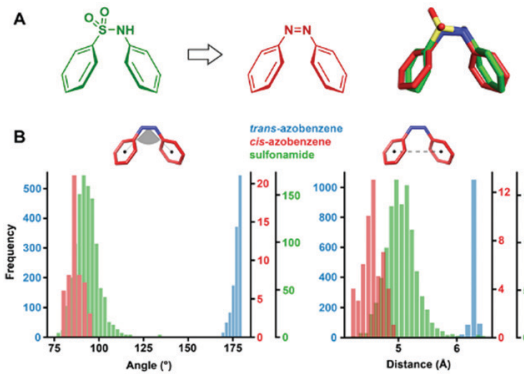

2.8.

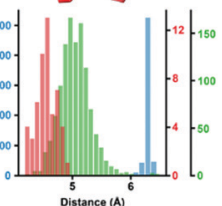

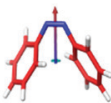

580

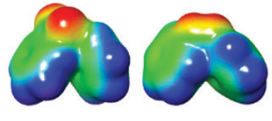

Fig. 1 (A). Biaryl sulfonamides as cisoid azosteres. (B). Structural similarity: distributions of centroid angles and ring distances in the CSD. (C). Electronic similarity: dipole moments and electrostatic potential surfaces, where red color represents max. negative potential and violet color represents max. positive potential.

similarity was explored by density functional theory (DFT) calculations (see ESI, $\dagger$ section S4.6 for details). As compared to trans-azobenzene, cis-azobenzene better approximates the dipole moment of BAS, as well as its electrostatic potential (ESP) surface (Fig. 1C). Finally, this moiety is widely used in medicinal chemistry ${ }^{20}$ (see ESI, $\dagger$ section S4.3 for details).

We hypothesized that the azologization of BAS would constitute a general strategy for designing cis-on ligands. However, the replacement of two hydrogen-bond acceptors and one donor in the $-\mathrm{SO}_{2} \mathrm{NH}$-linker part with two acceptors of the $-\mathrm{N}=\mathrm{N}-$ azo bond poses risks of loss in affinity to the target. To test our hypothesis and its limitations, two bioactive compounds containing the BAS moiety were identified and investigated by molecular modeling, photochemical evaluation and bioactivity studies. They were chosen because of their lownanomolar potency and the different roles of the sulfonamide moiety in binding to their biological targets. The first ligand (Sulf-1, Fig. 2A) is a nanomolar inhibitor of lipoproteinassociated phospholipase $2^{21}$ (Lp-PLA $A_{2}$, which is a potential therapeutic target. ${ }^{22}$ Here, the BAS moiety is buried and interacts deeply in the binding pocket. ${ }^{21}$ The second ligand (Sulf-2, Fig. 2B) is belinostat, ${ }^{23}$ an FDA-approved histone deacetylase (HDAC) inhibitor used in chemotherapy. In this case, the biaryl sulfonamide acts as a partially solvent-exposed linker that ensures optimal contacts of the capping group with the enzyme surface. $^{24}$

The starting point for the azologization of Sulf-1 was the crystal structure of its complex with Lp-PLA ${ }_{2}{ }^{25}$ The sulfonamide is involved in hydrogen bonds with Leu153, Ser273 and a water molecule occupying the oxyanion hole. ${ }^{26}$ Ligand binding was first explored in silico, using induced fit docking ${ }^{27}$ (IFD) to enable a certain degree of protein flexibility during the calculations. The docking poses suggested that cis-Azo-1 would be able to mimic the binding mode of the parent compound much better than its trans-isomer, albeit at the cost of the hydrogen bonding interactions (Fig. 2A). The stability of the docking poses over time was then evaluated through three replicas of $100 \mathrm{~ns}$ molecular dynamics (MD) simulations, which indicate that only trans-Azo-1 would unbind quickly from the protein during the simulation time, thus providing additional timedomain support for a preferred binding of the cis over the trans conformation. Moreover, the electronic similarity in terms of ESP surfaces and dipole moments found for the fragments was also confirmed for the entire ligands (see ESI, $\dagger$ Fig. S50 and S52).

Ligands Sulf-1 and Azo-1 were synthesized (see ESI, $\dagger$ section S1) and the photochemical properties of Azo-1 were investigated in Lp-PLA $\mathrm{P}_{2}$ assay aqueous buffer (Fig. 3A and B). Irradiation with $\lambda=365 \mathrm{~nm}$ light resulted in trans-cis photoisomerization (Fig. 3A), reaching a high photostationary state distribution (PSD) of $92 \%$ cis, typical of alkyl- and aryl-oxy substituted azobenzenes. ${ }^{28}$ Successive irradiation with $\lambda=420 \mathrm{~nm}$ light promoted cis-trans back-isomerization, reaching a PSD of $84 \%$ trans (see ESI, $\dagger$ Fig. S9). The photoswitch showed no signs of fatigue upon multiple cycles of photochemical isomerization in the presence of the reducing agent glutathione (Fig. 3B). Thermal cis-trans isomerization occurred on a time scale of days (see ESI, $\dagger$ Fig. S6 and S7), indicating high stability of cis isomer. Azo-1 showed good solubility in water (see ESI, $\dagger$ Fig. S11) up to the highest concentration used in the biological assay $(100 \mu \mathrm{M})$.

To assess the inhibitory potency of Sulf-1 and Azo-1 on Lp-PLA, we performed fluorescence-based competition assays ${ }^{29}$ (Fig. 3C).
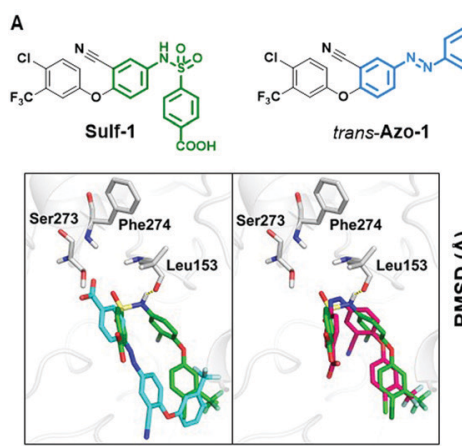
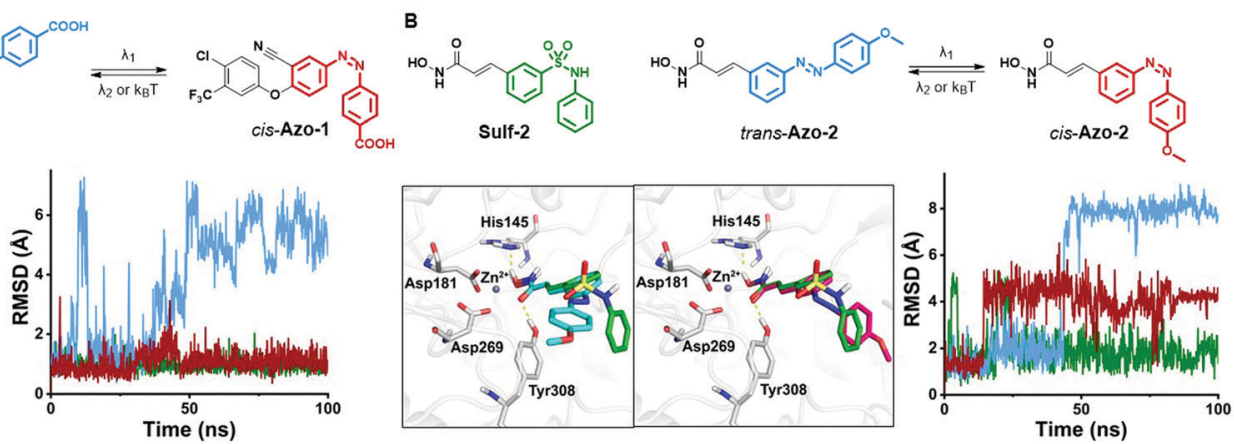

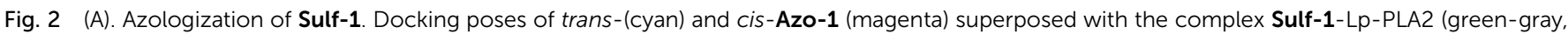

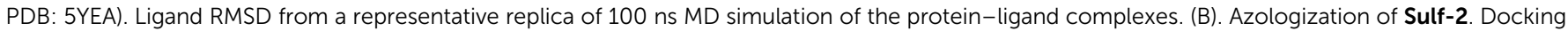

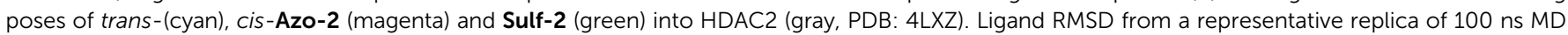
simulation of the protein-ligand complexes. For additional MD simulations, see ESI $\uparrow$ (section S4.5). 
A

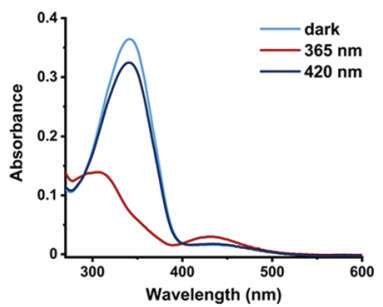

C

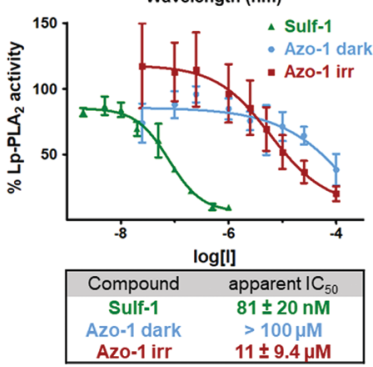

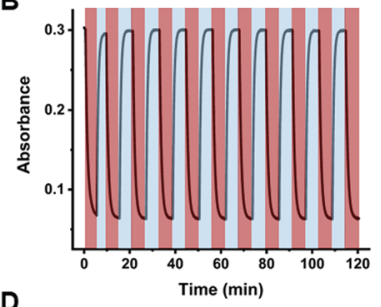

D

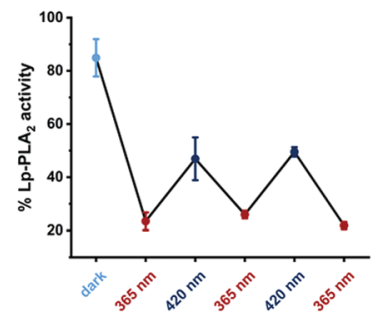

Fig. 3 Photochemical and pharmacological evaluation of Azo-1. (A). UV-Vis spectra of Azo-1 (20 $\mu$ M, 1\% DMSO in Lp-PLA 2 assay buffer) at the thermal equilibrium (light blue), PSS ${ }^{365 \mathrm{~nm}}$ (red) and PSS ${ }^{420} \mathrm{~nm}$ (dark blue). (B). Repeated photoisomerization of Azo-1 with $\lambda=365 \mathrm{~nm}$ and $420 \mathrm{~nm}$ light $(20 \mu \mathrm{M}, 1 \%$ DMSO in Lp-PLA 2 assay buffer, $10 \mathrm{mM}$ glutathione). (C). Dose-response curves for inhibitors Sulf-1/Azo-1 against $L p-P L A_{2}$ and obtained $I C_{50}$ values. (D). Reversible control of Lp-PLA 2 activity with Azo-1 $(25 \mu \mathrm{M})$.

The parent inhibitor Sulf-1 maintained its high reported activity in the nanomolar range, whereas the thermally adapted sample of Azo-1 showed very low activity. On the other hand, UV irradiation resulted in an >10-fold activation of Azo-1. Cycles of irradiation (Fig. 3D), starting from the thermally adapted sample and then alternating between the solutions that represent PSD at $\lambda=365 \mathrm{~nm}$ and $420 \mathrm{~nm}$ light, showed reversible behavior, indicating that the change in potency is caused by photoisomerization of Azo-1 and not by a light-induced irreversible process. Nevertheless, we observed an overall 100-fold decrease in activity of Azo-1 as compared to the parent Sulf-1, which might be due to the loss of crucial hydrogen bonds with the oxyanion hole of Lp-PLA ${ }_{2}{ }^{21}$

As a second test for our proposed azologization strategy, we chose belinostat $^{24}$ (Sulf-2), an inhibitor of HDACs used in clinical practice. In this case, a $p$-alkoxyazobenzene was employed, as it is known to show very high $\operatorname{PSDs}^{28}$ and appeared to be tolerated during previous SAR studies. ${ }^{23}$ Because of the available biological assay, we selected HDAC2 as protein target. Therefore, we needed to dock Sulf-2 into HDAC $2,{ }^{30}$ as the only crystal structure of the compound in the PDB is a complex with HDAC6. ${ }^{31}$ Although the two enzymes belong to different classes of HDACs, ${ }^{24}$ the inhibitor binding site is highly conserved (see ESI, $\dagger$ Fig. S27). The obtained docking pose revealed that the hydroxamic acid chelates the zinc cation and engages in hydrogen bonds with His145 and Tyr308, while the sulfonamide moiety is not involved in specific interactions with the enzyme (Fig. 2B). These contacts predicted for HDAC2 are in agreement with the binding mode observed experimentally with HDAC6 (see ESI, $\dagger$ Fig. S32 and S33). IFD poses of Azo-2 indicated that the cis-isomer of the ligand would adopt a similar binding mode and keep the original bent geometry. However, subsequent MD simulations
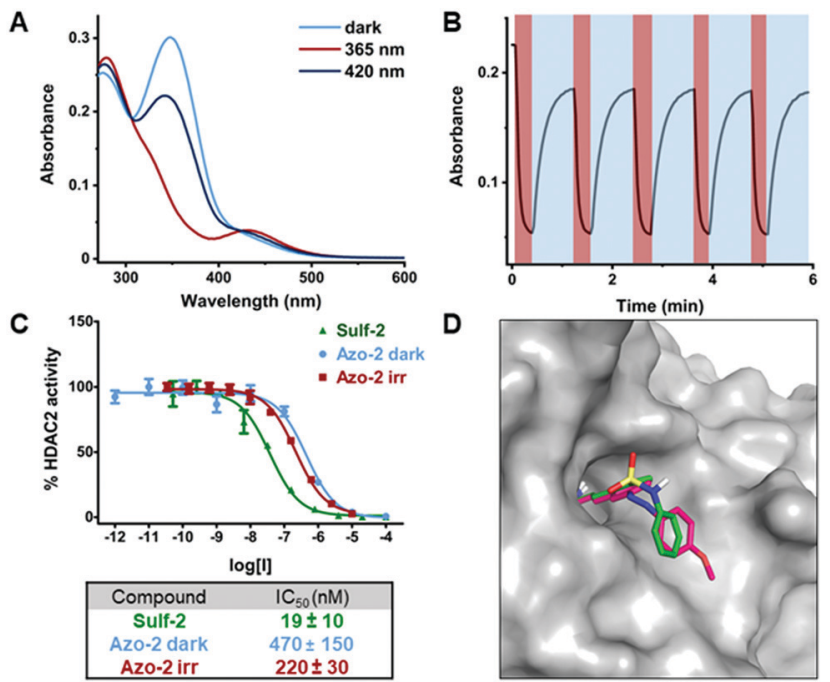

Fig. 4 Photochemical and pharmacological evaluation of Azo-2. (A). UV-Vis spectra of Azo-2 (20 $\mu \mathrm{M}, 1 \%$ DMSO in HDAC2 assay buffer) at the thermal equilibrium (light blue), PSS $365 \mathrm{~nm}$ (red) and PSS ${ }^{420 \mathrm{~nm}}$ (dark blue). (B). Photoisomerization of Azo-2 with $\lambda=365 \mathrm{~nm}$ and $420 \mathrm{~nm}$ light (20 $\mu$ M, 1\% DMSO in HDAC2 assay buffer, $10 \mathrm{mM}$ glutathione). (C). Doseresponse curves for inhibitors Sulf-2/Azo-2 against HDAC2 and obtained $I_{50}$ values. (D). Solvent exposure of Sulf-2 and cis-Azo-2 docked into HDAC2 (gray surface).

revealed that all the ligands were more flexible and explored larger portions of the protein surface, as the active site is exposed to the solvent (see Fig. 4D and ESI, $\dagger$ Fig. S36, S41, S46, S47). This predicted behavior suggests that the differences in activity between the ligands could be less pronounced than in the case of the Azo-1/Lp-PLA 2 system.

Photoisomerization of Azo-2 was achieved in HDAC2 assay buffer (Fig. 4A), reaching a high PSD of 95\% cis. Furthermore, the compound showed no fatigue after multiple cycles of photoisomerization under reducing conditions (Fig. 4B). HDAC2 inhibition assay confirmed the high potency of Sulf$2,{ }^{32}$ and much to our delight Azo-2 also showed activity in the sub-micromolar range (Fig. 4C). We found that irradiation with $\lambda=365 \mathrm{~nm}$ light resulted in a 2 -fold activation of Azo-2 (Fig. 4C), thereby showing the desired cis-on behavior of the photoswitchable inhibitor. Moreover, in this case we observed a smaller, 12-fold decrease in activity compared to the parent ligand. In contrast to the previous example, the azologized BAS moiety has a less essential role in binding, mainly providing the bent geometry for conformational control and does not contribute specific interactions. ${ }^{19,24}$

The inhibition data for Azo-1 and Azo-2 indicate that the pharmacophoric role of the BAS substructure influences the outcome of the azologization. When this moiety formed key interactions with buried residues of the oxyanion hole, ${ }^{25}$ its azostere Azo-1 suffered a larger loss of activity. Concurrently, a larger difference in activity between the photoisomers was achieved, suggesting that a cisoid geometry was strictly required for biological activity. However, when the sulfonamide moiety served only as a solvent-exposed linker to guarantee optimal contacts between the capping group and the target, ${ }^{24}$ the azolog 
showed a smaller loss of original activity. For Azo-2, the azologization involved a more peripheral region of the pharmacophore, while it did not affect the hydroxamic acid (i.e., the zinc binding domain). This substitution resulted in a less distinct difference between photoisomers.

In conclusion, we show here that BAS are promising cisoid azosteres for photopharmacology. While guidelines for transoid azosteres have been described and applied, ${ }^{4,33}$ the definition of criteria for the rational design of cis-on photoswitchable ligands is still lacking. To explore the requirements for molecular similarity with cis-azobenzene, we have analyzed the geometrical and electrostatic properties of two-atom-linked biaryl systems and selected the BAS motif based on its bent geometry and favorable dipole moment. We have demonstrated that biaryl sulfonamides are promising azosteres if they are considered for their preferred cisoid geometry, rather than being discarded for their poor similarity to trans-azobenzene. ${ }^{33}$ Since biaryl sulfonamides are common motifs in numerous ligands (see ESI, $\uparrow$ Table S3) for various biological targets (e.g., anti-apoptotic protein MCL- $1^{34}$ and bromodomain-containing protein $4^{35}$ ), we believe that they may provide a rich source of inspiration for photopharmacology. Azologization of cisoid substructures has the potential to guide the rational design of cis-on photoswitchable ligands.

Financial support from the EU Horizon 2020 program (ALERT co-fund No. 713482 for B. L. F.) and the Dutch Scientific Organization (VIDI grant nr. 723.014.001 for W. S., VIDI grant nr. 723.015.004 for M. D. W.) is kindly acknowledged. P. K. acknowledges dr. Romain Costil and Isabel Sieders (University of Groningen) for fruitful discussions, and the Center for Information Technology of the University of Groningen for their support and for providing access to the Peregrine HPC cluster.

\section{Conflicts of interest}

There are no conflicts to declare.

\section{Notes and references}

1 W. A. Velema, W. Szymanski and B. L. Feringa, J. Am. Chem. Soc., 2014, 136, 2178-2191.

2 M. M. Lerch, M. J. Hansen, G. M. van Dam, W. Szymanski and B. L. Feringa, Angew. Chem., Int. Ed., 2016, 55, 10978-10999.

3 M. W. H. Hoorens and W. Szymanski, Trends Biochem. Sci., 2018, 43, 567-575.

4 J. Broichhagen, J. A. Frank and D. Trauner, Acc. Chem. Res., 2015, 48, 1947-1960.

5 B. L. Feringa and W. R. Browne, Molecular Switches, Wiley-VCH, Weinheim, Germany, 2011.

6 A. A. Beharry and G. A. Woolley, Chem. Soc. Rev., 2011, 40, $4422-4437$.

7 M. Schoenberger, A. Damijonaitis, Z. Zhang, D. Nagel and D. Trauner, ACS Chem. Neurosci., 2014, 5, 514-518.

8 I. M. Welleman, M. W. H. Hoorens, B. L. Feringa, H. H. Boersma and W. Szymański, Chem. Sci., 2020, 11, 11672-11691.

9 C. Matera, A. M. J. Gomila, N. Camarero, M. Libergoli, C. Soler and P. Gorostiza, J. Am. Chem. Soc., 2018, 140, 15764-15773.
10 S. Pittolo, X. Gómez-Santacana, K. Eckelt, X. Rovira, J. Dalton, C. Goudet, J. P. Pin, A. Llobet, J. Giraldo, A. Llebaria and P. Gorostiza, Nat. Chem. Biol., 2014, 10, 813-815.

11 N. J. Hauwert, T. A. M. Mocking, D. Da Costa Pereira, K. Lion, Y. Huppelschoten, H. F. Vischer, I. J. P. De Esch, M. Wijtmans and R. Leurs, Angew. Chem., Int. Ed., 2019, 58, 4531-4535.

12 R. Siewertsen, H. Neumann, B. Buchheim-Stehn, R. Herges, C. Näther, F. Renth and F. Temps, J. Am. Chem. Soc., 2009, 131, 15594-15595.

13 J. B. Trads, K. Hüll, B. S. Matsuura, L. Laprell, T. Fehrentz, N. Görldt, K. A. Kozek, C. D. Weaver, N. Klöcker, D. M. Barber and D. Trauner, Angew. Chem., Int. Ed., 2019, 58, 15421-15428.

14 S. Li, N. Eleya and A. Staubitz, Org. Lett., 2020, 22, 1624-1627.

15 M. Borowiak, W. Nahaboo, M. Reynders, K. Nekolla, P. Jalinot, J. Hasserodt, M. Rehberg, M. Delattre, S. Zahler, A. Vollmar, D. Trauner and O. Thorn-Seshold, Cell, 2015, 162, 403-411.

16 M. W. H. Hoorens, M. E. Ourailidou, T. Rodat, P. E. van der Wouden, P. Kobauri, M. Kriegs, C. Peifer, B. L. Feringa, F. J. Dekker and W. Szymanski, Eur. J. Med. Chem., 2019, 179, 133-146.

17 K. A. Brameld, B. Kuhn, D. C. Reuter and M. Stahl, J. Chem. Inf. Model., 2008, 48, 1-24.

18 G. Schwertz, M. S. Frei, M. C. Witschel, M. Rottmann, U. Leartsakulpanich, P. Chitnumsub, A. Jaruwat, W. Ittarat, A. Schäfer, R. A. Aponte, N. Trapp, K. Mark, P. Chaiyen and F. Diederich, Chem. - Eur. J., 2017, 23, 14345-14357.

19 Y. Zheng, C. M. Tice and S. B. Singh, Bioorg. Med. Chem. Lett., 2017, 27, 2825-2837.

20 E. A. Ilardi, E. Vitaku and J. T. Njardarson, J. Med. Chem., 2014, 57, 2832-2842.

21 Q. Liu, F. Huang, X. Yuan, K. Wang, Y. Zou, J. Shen and Y. Xu, J. Med. Chem., 2017, 60, 10231-10244.

22 F. Huang, K. Wang and J. Shen, Med. Res. Rev., 2020, 40, 79-134.

23 P. W. Finn, M. Bandara, C. Butcher, A. Finn, R. Hollinshead, N. Khan, N. Law, S. Murthy, R. Romero, C. Watkins, V. Andrianov, R. M. Bokaldere, K. Dikovska, V. Gailite, E. Loza, I. Piskunova, I. Starchenkov, M. Vorona and I. Kalvinsh, Helv. Chim. Acta, 2005, 88, 1630-1657.

24 M. Mottamal, S. Zheng, T. L. Huang and G. Wang, Molecules, 2015, 20, 3898-3941.

25 P. Canning, B. A. Kenny, V. Prise, J. Glenn, M. H. Sarker, N. Hudson, M. Brandt, F. J. Lopez, D. Gale, P. J. Luthert, P. Adamson, P. Turowski and A. W. Stitt, Proc. Natl. Acad. Sci. U. S. A., 2016, 113, 7213-7218.

26 U. Samanta and B. J. Bahnson, J. Biol. Chem., 2008, 283, 31617-31624.

27 W. Sherman, T. Day, M. P. Jacobson, R. A. Friesner and R. Farid, J. Med. Chem., 2006, 49, 534-553.

28 W. Szymanski, M. E. Ourailidou, W. A. Velema, F. J. Dekker and B. L. Feringa, Chem. - Eur. J., 2015, 21, 16517-16524.

29 F. Huang, H. Hu, K. Wang, C. Peng, W. Xu, Y. Zhang, J. Gao, Y. Liu, H. Zhou, R. Huang, M. Li, J. Shen and Y. Xu, J. Med. Chem., 2020, 63, 7052-7065.

30 B. E. L. Lauffer, R. Mintzer, R. Fong, S. Mukund, C. Tam, I. Zilberleyb, B. Flicke, A. Ritscher, G. Fedorowicz, R. Vallero, D. F. Ortwine, J. Gunzner, Z. Modrusan, L. Neumann, C. M. Koth, J. S. Kaminker, C. E. Heise and P. Steiner, J. Biol. Chem., 2013, 288, 26926-26943.

31 Y. Hai and D. W. Christianson, Nat. Chem. Biol., 2016, 12, 741-747.

32 H. Y. Lee, C. Y. Chang, C. J. Su, H. L. Huang, S. Mehndiratta, Y. H. Chao, C. M. Hsu, S. Kumar, T. Y. Sung, Y. Z. Huang, Y. H. Li, C. R. Yang and J. P. Liou, Eur. J. Med. Chem., 2016, 122, 92-101.

33 J. Morstein, M. Awale, J. L. Reymond and D. Trauner, ACS Cent. Sci., 2019, 5, 607-618.

34 B. Follows, S. Fessler, T. Baumeister, A. M. Campbell, M. M. Zablocki, H. Li, D. Gotur, Z. Wang, X. Zheng, L. Molz, C. Nguyen, T. Herbertz, L. Wang and K. Bair, Bioorg. Med. Chem. Lett., 2019, 29, 2375-2382.

35 B. K. Allen, S. Mehta, S. W. J. Ember, J. Y. Zhu, E. Schönbrunn, N. G. Ayad and S. C. Schürer, ACS Omega, 2017, 2, 4760-4771. 